\title{
Uterine sarcomas: A clinicopathological analysis of 13 cases in a tertiary care centre
}

\author{
Pavithra P. ${ }^{1}$, Singh BK. ${ }^{2}$, Singh VK. ${ }^{3}$, Kairanna NV. ${ }^{4}$, Rao N. ${ }^{5}$, Shetty T. ${ }^{6}$, Padmapriya J. ${ }^{7}$
} DOI: https://doi.org/10.17511/jopm.2020.i02.05

1 Pavithra P., Associate Professor, Department of Pathology, Melaka Manipal Medical College, Manipal Academy of Higher Education, Manipal, Karnataka, India.

2* Brij Mohan Kumar Singh, Associate Professor, Department of Pathology, Kasturba Medical College, Manipal Academy of Higher Education, Manipal, Karnataka, India.

${ }^{3}$ Varun Kumar Singh, Assistant Professor, Department of Pathology, Melaka Manipal Medical College, Manipal Academy of Higher Education, Manipal, Karnataka, India.

4 Nikitha Valerina Kairanna, Assistant Professor, Department of Pathology, Kasturba Medical College, Manipal Academy of Higher Education, Manipal, Karnataka, India.

5 Namrata Rao, Assistant Professor, Department of Pathology, Melaka Manipal Medical College, Manipal Academy of Higher Education, Manipal, Karnataka, India.

6 Tanvi Shetty, Associate Professor, Department of Pathology, Melaka Manipal Medical College, Manipal Academy of Higher Education, Manipal, Karnataka, India.

7 Padmapriya J., Associate Professor, Department of Pathology, Kasturba Medical College, Manipal Academy of Higher Education, Manipal, Karnataka, India.

Introduction: Uterine sarcomas are rare tumors arising from mesodermal tissue of uterus which accounts for $1-3 \%$ of all female genital tract malignancies. They are characterized by rapid clinical progression and poor outcome. Materials and Methods: A retrospective review of clinical and pathological characteristics of patients with primary uterine sarcoma was done at Kasturba Hospital, Manipal between January 2013 and December 2018. Patients were staged using the 2018 FIGO histological classification for uterine cancer. Results: A total of 13 patients with uterine sarcoma were reviewed. All cases were multiparous women with the median age of presentation of 51.5 years (range: 40-80 years). Conclusion: Uterine sarcoma is an uncommon neoplasm with carcinosarcoma being the most common. The prognosis of uterine sarcomas depends on the histological subtype, grade and stage of the tumor at diagnosis.

Keywords: Adenosarcoma, Carcinosarcoma, Leiomyosarcoma, PEComa, Uterine sarcomas

\section{Corresponding Author}

Brij Mohan Kumar Singh, Associate Professor, Department of Pathology, Kasturba Medical College, Manipal Academy of Higher Education, Manipal, Karnataka, India.

Email: brij.singh@manipal.edu
How to Cite this Article

Pavithra $P$, Singh BMK, Singh VK, Kairanna NV, Rao N, Shetty T, Padmapriya J. Uterine sarcomas: A clinicopathological analysis of 13 cases in a tertiary care centre. Trop J Pathol Microbiol. 2020;6(2):139145. Available From https://pathology.medresearch .in/index.php/jopm/article/view/413
To Browse

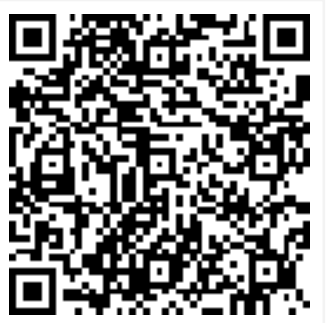

Manuscript Received 10-01-2020

Conflict of Interest No
Review Round 1 20-01-2020

Funding

$\mathrm{Nil}$
Review Round 2 28-01-2020

Ethical Approval Yes
Review Round 3

Plagiarism X-checker $17 \%$
Accepted 03-02-2020

Note

(c) 2020 by Pavithra P., Brij Mohan Kumar Singh, Varun Kumar Singh, Nikitha Valerina Kairanna, Namrata Rao, Tanvi Shetty, Padmapriya J. and Published by Siddharth Health Research and Social Welfare Society. This is an Open Access article licensed 


\section{Introduction}

Uterine sarcomas are uncommon tumors with a prevalence of $1-3 \%$ of all female genital tract malignancies and $3-7 \%$ of uterine cancers [1]. These tumors arise from mesodermal tissue of the uterus. A myriad of entities are included under the umbrella of uterine sarcomas, the commonest being leiomyosarcoma (LMS) (40\%) which arises from myometrium, carcinosarcoma (CS) (40\%) and endometrial stromal sarcoma (ESS) (10-15\%) which arise from the endometrium, adenosarcoma (AS) (0-5\%) and the rarest being perivascular epithelioid cell tumor (PEComa). Gross pathological features like tumor size, pelvic and other abdominal organ involvement hold prognostic significance in cases of LMS and ESS; however, in cases of AS myometrial invasion is the most significant gross feature. In the case of CS, the gross features are similar to those of carcinomas of endometrial origin. Histopathological examination is an essential feature in the diagnosis of these entities. LMS and ESS are spindle cell tumors, while CS and AS are biphasic and show both epithelial and mesenchymal differentiation. PEComas are a unique entity as they are of mesenchymal origin but show an epithelioid morphology that mimics a carcinoma. Ancillary diagnostic techniques like immunohistochemistry are usually employed for a definitive diagnosis. The common immunohistochemical markers used include smooth muscle actin for LMS, CD10 for ESS, vimentin for mesenchymal differentiation and cytokeratin to detect epithelial elements in a biphasic tumor. HMB45 is frequently used in the diagnosis of PEComas as they co-express myoid and melanocytic markers. In comparison to other epithelial uterine malignancies, uterine sarcomas pose a dreaded outcome due to their aggressive behavior [2,3]. Patients generally present with abnormal uterine bleeding, abdominal or pelvic mass and pain. Surgery is the primary modality of treatment and helps in staging, histological subtyping, grading of the malignancy. International Federation of Gynecology and Obstetrics (FIGO) criteria of 1988 for endometrial carcinoma was used for quite some time for staging of uterine sarcomas in spite of the difference in their behavior. In 2018, a specific FIGO staging system for sarcomas was devised. LMS, ESS, AS and PEComas are graded along the lines of sarcoma, while CS is graded and staged on the lines of endometrial carcinoma. $[4,5]$ and recurrence of these sarcomas are seen frequently over a period of time [5]. The patient
Receives adjuvant chemo and radiotherapy to reduce the incidence of recurrence, but they do not aid in improving the overall survival $[6,7]$. Due to the rarity and pathological diversity of these tumors, there is no consensus on the optimal management. This study aims to analyze the clinicopathological features of uterine sarcoma and their treatment outcome.

\section{Materials and Methods}

Setting: Single-center retrospective study

Duration and type of study: A retrospective study was conducted at the department of pathology, Kasturba hospital, Manipal, over a period of six years between January 2013 and December 2018.

Sampling methods: Data retrieved from the coded departmental archives.

Inclusion criteria: All patients with a pathological diagnosis of uterine sarcoma were included in the study.

Exclusion criteria: All epithelial uterine malignancies were excluded.

Ethical clearance: Institutional ethical committee clearance was obtained prior to the study.

Data collection procedure: Archive of the department of pathology was searched for uterine sarcoma The patients' medical records were reviewed for clinical history, tumor characteristics, treatment, and follow-up data. The point of the last contact was considered for follow up data.

Data analysis: All the histopathology and immunohistochemistry slides were reviewed, and the staging of the disease was determined retrospectively. In cases where staging laparotomy was not performed, the stage was assigned based on the available pathologic findings.

Staging system: Staging was done according to the International Federation of Gynecology and Obstetrics (FIGO) 2018 criteria.

\section{Results}

A total of 13 cases of uterine sarcoma were reviewed. The median age of presentation was 51.5 years (Range: $40-80$ years). All the cases were multiparous females. CS was the most common histological type $(38.46 \%)$ followed by ESS $(30.76 \%)$. Other histological types include adenosarcoma, leiomyosarcoma, and PEComa 
(Figure 1).

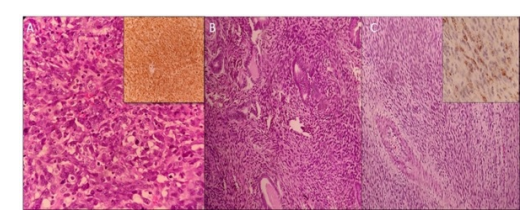

Figure 1. A) Leiomyosarcoma- malignant spindle cells in confluent clusters with increased mitosis ( $H$ and $E, 400 x$ ). The tumor cells are positive for smooth muscle actin (inset). B) Adenosarcoma- malignant endometrial glands surrounded by spindle cell sarcomatous component ( $H$ and $E, 400 x$ ). C) PEComa- perivascular epithelioid cells with bland nuclear features ( $H$ and $E, 100 x$ ). The tumor cells are positive for HMB45 (inset).

Post-menopausal bleeding and abdominal pain were the most common clinical presentation. Ten cases
Underwent total abdominal hysterectomy with bilateral salpingo-oophorectomy (TAH+BSO). Two patients underwent total hysterectomy for clinical diagnosis of uterine fibroid, which was diagnosed to be LMS in one and PEComa in the other. The patient with LMS further underwent staging laparotomy with metastatic deposits in the pelvic nodes and hence was upgraded to FIGO stage IIIc. Most of the patients in this study were in FIGO stage I at presentation. The demographic, clinical and pathological features are summarized in Tables 1 and 2. On immunohistochemistry, all CS cases expressed cytokeratin (CK) and epithelial membrane antigen (EMA) in the epithelial component and vimentin in the stromal component. CD10 was found to be a consistent marker for AS and ESS. Hormone receptors (estrogen and progesterone receptor) were positive in AS, ESS (Low grade), and LMS. HMB45 was positive in PEComa.

Table-1: Demographic and clinico-pathological characteristics of the patients with uterine sarcomas

\begin{tabular}{|c|c|c|c|c|c|}
\hline Parameters & Carcinosarcoma $(\mathrm{n}=5)$ & Endometrial stromal sarcoma $(n=4)$ & Adenosarcoma ( $n=2$ & Leiomyosarcoma ( $\mathrm{n=1}$ & PEComa $(n=1)$ \\
\hline Age(years)(Mean \pm SD) & $63.2 \pm 7.5$ & $41.75 \pm 20.1$ & $59.5 \pm 21.9$ & 43 & 43 \\
\hline Parity (median) & 3 & 2 & 2 & 4 & 3 \\
\hline \multicolumn{6}{|l|}{ Medical Illness } \\
\hline Diabetes & 2 & 0 & 1 & 1 & 0 \\
\hline Hypertension & 2 & 1 & 0 & 0 & 0 \\
\hline Pre-operative biopsy & 0 & 1 & 0 & 3 & Nil \\
\hline \multicolumn{6}{|l|}{ Myometrial Invasion } \\
\hline None & 2 & 4 & 2 & 1 & 1 \\
\hline$<50 \%$ & 2 & 0 & 0 & 0 & 0 \\
\hline$>50 \%$ & 1 & 0 & 0 & 0 & 0 \\
\hline Cervical involvement & Nil & Nil & Nil & Nil & Nil \\
\hline \multicolumn{6}{|l|}{ Lymph Node } \\
\hline Negative & 2 & 4 & 1 & 1 & 1 \\
\hline Positive & 1 & 0 & 1 & 0 & 0 \\
\hline Not Done & 2 & 0 & 0 & 0 & 0 \\
\hline Lymphovascular invasion & & Nil & Nil & Nil & Nil \\
\hline \multicolumn{6}{|l|}{ Pelvic washing } \\
\hline Negative & 3 & 3 & 1 & 1 & 1 \\
\hline Positive & 1 & 0 & 0 & 0 & 0 \\
\hline Not done & 1 & 1 & 1 & 0 & 0 \\
\hline \multicolumn{6}{|l|}{ Omentum involvement } \\
\hline Negative & 2 & 3 & 1 & 1 & 1 \\
\hline Positive & 1 & 0 & 0 & 0 & 0 \\
\hline Not done & 2 & 1 & 1 & 0 & 0 \\
\hline
\end{tabular}

Table-2: Staging and Treatment of patients with uterine sarcomas.

\begin{tabular}{|l|l|l|l|l|l|l|}
\hline Parameters & Carcinosarc-oma $(n=5)$ & Endometrial stromal sarcoma $(n=4)$ & Adenosarco-ma $(n=2)$ & Leiomyosar-coma $(n=1)$ & PeCoMA \\
\hline FIGO & 4 & 3 & 2 & 0 & 1 \\
\hline I & 4
\end{tabular}




\begin{tabular}{|c|c|c|c|c|c|}
\hline II & 1 & 1 & 0 & 0 & 0 \\
\hline III & 0 & 0 & 0 & 1 & 0 \\
\hline IV & 0 & 0 & 0 & 0 & 0 \\
\hline \multicolumn{6}{|l|}{ Surgical Procedure } \\
\hline TAH+BSO & 4 & 4 & 2 & TAH + right & 1 \\
\hline TAH+BSO+STAGING & 1 & 0 & 0 & \multirow[t]{2}{*}{ salphingo-ophrectomy } & 0 \\
\hline TAH+BSO+ DEBULKING & 0 & 0 & 0 & & 0 \\
\hline Adjuvant chemotherapy & 2 & 2 & 0 & 0 & 0 \\
\hline Adjuvant radiotherapy & 2 & 2 & 0 & 0 & 0 \\
\hline Mean follow up (months) & 9 & 4 & 6 & 12 & 4 \\
\hline
\end{tabular}

\section{Discussion}

Uterine sarcoma is a heterogeneous malignancy, with much debate on their subtyping and management. The age at presentation differs based on the histologic subtype; LMS often occurs in the perimenopausal age group while CS and ESS occur in the postmenopausal age group [8]. Hysterectomy and BSO are the preferred treatment of choice for uterine sarcomas [9]. Carcinosarcoma, as the name suggests, is a tumor that shows epithelial and mesenchymal differentiation on histology and it comprises $<5 \%$ of all uterine tumors. It is common in the age group of the 6th- 7th decade [10] [11]. Around 5- $30 \%$ of these patients had undergone prior pelvic irradiation with a latency of 14 years post-radiation. Grossly, these tumors present as a solitary polypoid mass projecting into the uterine cavity with deep infiltration of the myometrium. Based on the sarcomatous component, they are classified as homologous and heterologous type. The carcinomatous component is usually of endometrioid, serous or clear cell type [12]. In the present study, five cases of CS were identified with the mean age of presentation of $63.2 \pm 7.5$ years. One case had a history of pelvic irradiation following a malignancy. The myometrial invasion was seen in 3 cases. The epithelial component was endometrioid in 3 and serous in 2 cases. The sarcomatous component was homologous type and it was a spindle cell morphology. On immunohistochemistry, all cases were positive for panCK, EMA and vimentin. The tumor can show a variable proportion of epithelial and sarcomatous elements. The presence of a single predominant population necessitates extensive sampling of the tumor in order to identify the other component $[10,13]$. ESS is a very rare malignant neoplasms that comprise $0.2 \%$ of all uterine malignancies and $10 \%$ of all uterine sarcomas with unknown pathogenesis [14]. The majority of the patients are premenopausal with
A mean age of occurrence of $42-58$ years $[14,15]$. Exposure to tamoxifen, unopposed estrogen, and polycystic ovarian disease are implicated risk factors for ESS. They commonly present with abnormal uterine bleeding, abdominal mass, pelvic pain and dysmenorrhea [15-17]. In the present study, four cases of ESS were identified. The mean age of presentation was $41.75 \pm 20.1$ years. Abnormal uterine bleeding was seen in all four cases while two presented with an abdominal mass. Uterine curettage can be helpful in pre-operative diagnostic modality; however, when the lesion is completely intra-myometrial, scrapings may not yield a definitive diagnosis [16]. In the present study, a preoperative diagnosis of curettage material was made in one case. The term ESS is restricted to neoplasms earlier called low-grade ESS and the high-grade tumors are now called undifferentiated endometrial sarcomas. ESS grossly presents as single or multiple nodules with poor demarcation and cystic degeneration [17]. The present study showed multiple solid cystic masses in all four cases. Strong and diffuse positivity for CD10 helps differentiation of ESS from leiomyoma, which was seen in all four cases in the present series. LMS is a very rare uterine malignancy with an annual incidence of 0.5 to 3.3 per 100,000 women [18]. They are aggressive smooth muscle malignancies and present mostly as solitary lesions. They are usually not associated with leiomyoma although the malignant transformation of the myoma is a rare event [19]. Out of two cases of the LMS in the present study, one case was clinically diagnosed as leiomyoma. Both the cases presented with vaginal bleeding and pain abdomen with intrauterine solitary lesion identified on radiology. These clinical findings were seen in all cases of LMS as documented in the literature [20]. Preoperative screening does not play any role in the diagnosis or staging of LMS as there is marked histological variation seen within the tumor. Even though the 
Treatment of choice is surgical in FIGO stage I and II, the recurrence rate is up to $70 \%$ in these cases [21]. There is no beneficial effect of surgical treatment when the disease is extending beyond the peritoneal cavity [22]. In the current study, both the patients had undergone TAH with BSO in one and right salpingo-oophorectomy in the other. They were in FIGO stage I and did not show any features of recurrence after a close follows up of 12 months. No adjuvant chemotherapy or radiotherapy was administered. $70 \%$ of the uterine LMS is usually diagnosed in FIGO stage I and II [23]. Recent reports suggest that radical hysterectomy serves a favorable prognosis. The role of BSO in a case of uterine sarcoma is controversial. Various studies reported that adnexectomy improves the prognosis of the patients [24]. Adenosarcomas are low-grade malignant tumors characterized by malignant stromal and benign epithelial elements and are known for local recurrence. They are classified into homologous type (histologically benign epithelioid component) and heterologous (carcinomatous epithelioid component) [25].

Both cases described in the present study were of homologous type. It usually arises in the endometrium or as a polypoidal growth from the cervix, the myometrium, fallopian tube, and ovary. Uterine AS mostly presents with genital bleeding. Both of our described cases presented in their 6th decade of life. Histologically they were in FIGO stage I with no myometrial invasion and no involvement of either nodes or lymphovascular invasion and had undergone TAH + BSO as both cases were in the postmenopausal period, whereas in premenopausal patients TAH without BSO would be an option [26]. In cases of uterine AS, unfavorable prognostic factors are sarcomatous overgrowth, deep myometrial invasion, and the presence of heterologous elements with extrauterine spread [27].

The present cases were in close follow up for six months with no signs of recurrence. PEComas of gynecologic origin are rare and immunophenotypically resembles the perivascular epithelioid cells and clear cell sugar tumor of the lung [28]. They are seen most commonly in middleaged women with a median age of presentation of 38 years. The hallmark of PEComas is co-expression of melanocytic and smooth muscle markers $[29,30]$. The present case is of a 43-year-old female with abdominal mass and pain. The patient underwent TAH BASO and histopathology showed an epithelioid
Cell tumor with immunohistochemical positivity for SMA and HMB45, hence it was reported as uterine PEComa with FIGO stage I. The patient was disease-free for an available follow-up period of four months. Owing to the rarity of the entity, clear prognostic factors and outcome have not been documented for uterine PEComas. The present case adds to the existing literature of this rare entity. The management of uterine sarcomas includes a composite approach with surgical and chemo/radiotherapy. The commonly followed protocol includes a surgical resection which helps in the staging of the malignancy. Adjuvant chemotherapy and radiotherapy are implicated only in a handful of cases. Such a multimodality approach has shown to yield a significantly longer median disease-specific survival; however, the benefits are marred by clinical heterogeneity of the malignancy and lacks extensive documentation. Systemic chemotherapy has been found to be useful in cases of extensive metastatic disease [10]. In the present study, surgical resection was done in all cases. Adjuvant chemotherapy and radiotherapy were given in four cases. All cases were followed up clinically and radiologically with no recurrence over a maximum period of 12 months.

Limitations: Most of our patients in the present study were lost to follow up due to which the study has not been able to yield information regarding survival rate and recurrence rate.

\section{Conclusion}

Uterine sarcomas are rare tumors with some of the included entities only reported as isolated case reports. Much work has been done on LMS and ESS, however, CS, AS, and PEComas still remain shrouded in uncertainty in terms of diagnostic features, optimal management, and outcome. The prognosis of these sarcomas depends on the histological subtype, grade and stage of the tumor at diagnosis. Hysterectomy has been the standard surgical treatment.

\section{What does the current study add to the existing knowledge?}

The present series adds to the clinical and pathological features of these rare neoplasms affecting the uterine corpus. The role of adjuvant radiotherapy is still debatable and is said to help in better local control and reduction of the recurrence rate which was also evident in the present study. 


\section{Author's contribution}

Dr. Pavithra P: Concept, design, data analysis

Dr. Brij Mohan Kumar Singh: Concept, design, data analysis

Dr. Varun Kumar Singh: Data acquisition, data analysis

Dr. Nikitha Valerina Kairanna: Data acquisition

Dr. Namrata Rao: Data acquisition

Dr. Tanvi Shetty: Data acquisition

Dr. Padmapriya J: Data acquisition

\section{Reference}

01. Major F J, Blessing J A, Silverberg S G, Morrow C P, Creasman W T, Curie J L, Yordan E BMF. Prognostic factors in early stage uterine sarcoma- a Gynecologic Oncology Group study. Cancer. 1993;71(4)1702-1709.

[Crossref]

02. Dinh TA, Oliva EA, Fuller AF Jr, Lee H GA. The treatment of uterine leiomyosarcoma Results from a 10-year experience (1990-1999) at the Massachusetts General Hospital. Gynecol Oncol. 2004;92(2)648-652.

doi:[Article:https://doi.org/10.1016/j.ygyno.2003. 10.044][Crossref]

03. Kelly KLJ, Craighead PS. Characteristics and management of uterine sarcoma patients treated at the Tom Baker Cancer Centre. Int J Gynecol Cancer. 2005;15(1)132-139. doi:[Article:https://doi.org/10.1111/j.1048-891x. 2005.15014.x][Crossref]

04. Schick U, Bolukbasi Y, Thariat J, Abdah-Bortnyak $R$, Kuten A, Igdem $S$, et al. Outcome and prognostic factors in endometrial stromal tumors- A rare cancer network study. Int ] Radiat Oncol Biol Phys. 2012;82(5)e757-e763. doi:[Article:https://doi.org/10.1016/j.ijrobp.2011. $11.005]$ [Crossref]

05. Giuntoli RL, Metzinger DS, DiMarco CS, Cha SS, Sloan JA, Keeney GL, et al. Retrospective review of 208 patients with leiomyosarcoma of the uterus- Prognostic indicators, surgical management, and adjuvant therapy. Gynecol Oncol. 2003;89(3)460-469.

doi: [Article:https://doi.org/10.1016/s00908258(03)00137-9][Crossref]
04. Livi L, Paiar F, Shah N, Blake P, Villanucci A, Amunni $G$, et al. Uterine sarcoma- Twenty-seven years of experience. Int J Radiat Oncol Biol Phys. 2003;57(5)1366-1373.

doi:[Article:https://doi.org/10.1016/S0360-3016(03) 00750-8][Crossref]

05. Iwasa $Y$, Haga H, Konishi I, Kobashi Y, Higuchi K, Katsuyama $E$, et al. Prognostic factors in uterine carcinosarcoma- A clinicopathologic study of 25 patients. Cancer. 1998;82(3)512-519.

[Crossref]

06. Sutton G, Brunetto VL, Kilgore L, Soper JT, McGehee $R$, Olt $G$, et al. A phase III trial of ifosfamide with or without cisplatin in carcinosarcoma of the uterus- A Gynecologic Oncology Group study. Gynecol Oncol. $2000 ; 79(2) 147-153$.

doi:[Article:https://doi.org/10.1006/gyno.2000. 6001][Crossref]

07. Kanthan R, Senger J-L. Uterine Carcinosarcomas (Malignant Mixed Müllerian Tumours)- A Review with Special Emphasis on the Controversies in Management. Obstet Gynecol Int. 2011.

doi: [Article:https://doi.org/10.1155/2011/470795] [Crossref]

08. Gadducci A, Cosio S, Romanini A, Genazzani AR. The management of patients with uterine sarcoma- A debated clinical challenge. Crit Rev Oncol Hematol. 2008;65(2)129-142.

doi:[Article:https://doi.org/10.1016/j.critrevonc. 2007.06.011][Crossref]

09. Sait HK, Anfinan NM, Sayed ME El, Alkhayyat SS, Ghanem AT, Abayazid RM, et al. Uterine sarcoma. Saudi Med J. 2014;35(10)1215-1222. [Crossref]

10. Banik T, Halder D, Gupta N DP. Malignant Mixed Mullerian Tumor of the Uterus- Diagnosis of a Case by Fine-Needle Aspiration Cytology and Review of Literature. Diagn Cytopathol. $2011 ; 40(7) 635-657$.

doi: [Article:https://doi.org/10.1002/dc.21681] [Crossref]

11. Ramondetta LM, Burke TW, Jhingran A, Schmandt R, Bevers MW, Wolf JK, et al. A phase II trial of cisplatin, ifosfamide, and mesna in patients with advanced or recurrent uterine malignant mixed müllerian tumors with evaluation of potential molecular targets. Gynecol Oncol. 2003;90(3)529-536. doi:[Article:https://doi.org/10.1016/s0090-8258(03) 00332-9][Crossref] 
11. D'Angelo E, Prat J. Uterine sarcomas- A review. Gynecol Oncol [Internet]. 2010;116(1)131-139. doi:

[Article:http://dx.doi.org/10.1016/j.ygyno.2009.09.0 23][Crossref]

12. Puliyath G, Nair MK. Endometrial stromal sarcoma- A review of the literature. Indian J Med Paediatr Oncol. 2012;33(1)1-6.

[Crossref]

13. Ashraf-Ganjoei T, Behtash N, Shariat M, Mosavi A. Low grade endometrial stromal sarcoma of uterine corpus, a clinico-pathological and survey study in 14 cases. World J Surg Oncol. 2006;4;2-6.

doi: [Article:https://doi.org/10.1186/1477-7819-450][Crossref]

14. Park JY, Kim DY, Suh DS, Kim JH, Kim YM, Kim $Y T$, et al. Prognostic factors and treatment outcomes of patients with uterine sarcomaAnalysis of 127 patients at a single institution, 1989-2007. J Cancer Res Clin Oncol. 2008;134(12)1277-1287.

doi: [Article:https://doi.org/10.1007/s00432-0080422-2][Crossref]

15. Harter P, El-Khalfaoui K, Heitz F, Du Bois A. Operative and conservative treatment of uterine sarcomas. Geburtshilfe Frauenheilkd. $2014 ; 74(3) 267-270$.

doi: [Article:https://dx.doi.org/10.1055\%2Fs-00341368204][Crossref]

16. Cliuang JT, Van Velden, DJJ GJB. Carcinosarcoma and mixed mesodermal tumors of the uterine corpus. Obs Gynecol. 1970;35(5)766-780.

[Crossref]

17. Hyman DM, Grisham RN, Hensley ML. Management of advanced uterine leiomyosarcoma. Curr Opin Oncol. 2014;26(4)422-427. doi:[Article:https://doi.org/10.1097/CCO.0000000 000000094][Crossref]

18. Indraccolo $U$, Luchetti $G$ IS. Malignant transformation of uterine leiomyomata. Eur J Gynaecol Oncol. 2008;29(5)543-544. [Crossref]

19. Tinelli A. Uterine Leiomyosarcoma- A Rare Cancer Much as Complicated to Prevent, Diagnose and Treat. Austin J Obs Gynecol. 2014;1(5)3-5.

[Crossref]
19. Gurram MK, Pulivarthi S, McGary CT, Defillo A. Brain and multiorgan metastases from uterine leiomyosarcoma. Tumori. 2014;100(1)8-13.

doi: [Article:https://doi.org/10.1700/1430.15829] [Crossref]

20. Sinha A, Phukan JP, Sengupta S, Guha P. Mullerian adenosarcoma of uterus with sarcomatous overgrowth and heterologous component associated with stromal deposit in omentum- A case report and review of the literature. Case Rep Med. 2012.

doi: [Article:https://doi.org/10.1155/2012/820378] [Crossref]

21. Morice P, Rodrigues A, Pautier P, Rey A, Camatte $S$ AD. Surgery for uterine sarcoma- review of literature and recommendation for the standard surgical procedure. Gynecol Obs Fertil. 2003;32(2)147-150.

doi: [Article:http://doi.org/10.1016/s12979589(03)00061-4][Crossref]

22. Folpe $A L$, Mentzel $T$, Lehr $H$, Fisher $C$, Balzer $B L$, Weiss SW. Perivascular Epithelioid Cell Neoplasms of Soft Tissue and Gynecologic Origin A Clinicopathologic Study of 26 Cases and Review of the Literature. Am J Surg Pathol. 2005;29(12)1558-1575.

doi:[Article:https://doi.org/10.1097/01.pas.00001

73232.22117.37][Crossref]

23. Berkowitz RS GD. Uterine cancer, In- Berek JS HNW\& W, editor. Practical Gynecologic Oncology, 4th ed. 2005,p- 431-432.

[Crossref]

24. Kitazume $Y$, Shibuya $H$, Sekiguchi $M$, Nakaminato S, Taura S, Sato $S$, et al. Adenosarcoma of the uterus- A case report. Japanese J Clin Radiol. 2010;55(13)1773-1777. doi: [Article:https://dx.doi.org/10.5795/jjscc.20.149] [Crossref]

25. Çelik H, Kefeli M, Çetinkaya M, Yıldız L. Perivascular epithelioid cell tumor (PEComa) of the uterine cervix in a patient with tuberous sclerosis complex- A literature review. Turk Patoloji Derg. 2018;34(1)82-86. doi:[Article:https://doi.org/10.5146/tjpath.2014. 01274][Crossref]

26. Fukunaga M. Perivascular epithelioid cell tumor of the uterus- Report of four cases. Int J Gynecol Pathol. 2005;24(4)341-346. doi:[Article:https://doi.org/10.1097/01.pgp.000016 8515.83557.89][Crossref] 